\title{
Psychoactive substances related to the deaths
}

\author{
Sidlo J, Mlynar J, Kuruc R, Ocko P, Valuch J \\ Institute of Forensic Medicine, Faculty of Medicine, Comenius University and Health Care Surveillance Authority, \\ Bratislava, Slovakia.sidlo45@gmail.com
}

\begin{abstract}
The study included all deceased who died in relation to psychoactive substances abuse in the regions of Bratislava and Trnava city districts within a two-year period of the years 2007-2008. All the cases were autopsied by the standard method completed with toxicological analyses. There were reported total 84 fatalities related to psychoactive substances. From this amount, 38 cases $(45 \%)$ belong to the group of direct deaths (poisonings) and 46 cases (55\%) to the group with other cause of death. Out of the total number, $83 \%$ were related to men and $17 \%$ to women. More than $50 \%$ of the cases were related to the age up to 34 years. In the group of poisonings, the most frequently detected substances were opioids themselves or in combination with other substances in $53 \%$. In the group of the indirect deaths, the most frequent category of death was suicide in $39 \%$. The most frequently detected psychoactive substances were central nervous system stimulants in $40 \%$ of cases (Tab. 6, Fig. 1, Ref. 16). Full Text in free PDF www.bmj.sk. Key words: psychoactive substances related deaths, autopsy, toxicology, monitoring.
\end{abstract}

Abuse of drugs and psychoactive substances commonly used mainly for the purpose of mental affections treatment but also for the treatment of other sickly states represents a big health and health-service as well as a social problem. Its worst consequence is death of a human being. Monitoring of deaths related to psychoactive substances represents a useful indicator of trends in their abuse (1). Besides that, monitoring of drugs and psychoactive substances related deaths serves as one of the bases for the creation of anti-drug programs and politics on national and international standard as well.

The European Union member states are obliged to collect data on the psychoactive substances related deaths and provide them to the European Monitoring Centre for Drugs and Drug Addiction (EMCDDA) in Lisbon. According to the EMCDDA method, the psychoactive substances related deaths are divided into two groups: direct and indirect. The "direct deaths" are defined as the deaths caused directly by consumption of abused drugs. They occur generally shortly after a consumption of the substance(s). Those cases are further divided in relation to participation of the substances on death (Tab. 1). In the group of so called "indirect deaths" the causes of death are other than poisoning. Within the group the cases are sorted according to the category of death (Tab. 2) (2).

Recently, in our scientific literature, there were published reduced size studies related only to traffic accidents deaths caused by psychoactive substances affection $(3,4)$.

Institute of Forensic Medicine, Faculty of Medicine, Comenius University and Health Care Surveillance Authority, Bratislava, Slovakia

Address for correspondence: J. Sidlo, MD, PhD, Inst of Forensic Medicine, School of Medicine, Comenius University and Health Care Surveillance Authority, Antolska 11, SK-857 01 Bratislava, Slovakia. Phone: +421.2.59357264, Fax: +421.2.63531990

Acknowledgement: The study was presented on the scientific meeting of Bratislava Physicians Guild of the Slovak Medical Association on March 22, 2010.
The objective of our study was to present the most up-to-date complex review of all categories of deaths related to the psychoactive substances other than alcohol.

\section{Patients}

The study included all deceased who died in relation to psychoactive substances abuse in the regions of Bratislava and Trnava city districts within a two-year period of the years 2007-2008. All cases were autopsied by the standard method at the Institute of Forensic Medicine of Health Care Surveillance Authority and up to August 2007 also at the Institute of Forensic Medicine of Medical School of Comenius University in Bratislava. At the time of the study, the zone of both institutions had included about 1.17 million inhabitants, which represented approximately one fifth of the total population in Slovakia. According to the National Decree - Act No. 581, §48, all psychoactive substances related fatalities should undergo a complete medicolegal autopsy performed by a medical examiner (5). All available police records, hospital records and necropsy protocols, including histopathological and toxicological analyses, were scrutinized. The cases were divided into two groups - direct deaths and indirect deaths according to the above mentioned criteria. The data was analyzed according to the cause of death, sex, age and detected psychoactive substances. The results of analysis were compared to data acquired from other regions of Slovakia within the scope of monitoring deaths by the Health Care Surveillance Authority for the National Monitoring Centre for Drugs.

\section{Methods}

For histopathological examinations, samples of organs and tissues were taken, fixed in formalin, processed using a routine 
Tab. 1. Classification of cases according the substance(s) detected by poisoning.

Cause of death

Poisoning by opioids only (excluding methadone)

Poisoning by methadone only

Poisoning by (poly)substances including opioids

Poisoning by (poly)substances excluding opioids

Poisoning by psychoactive medicines

Poisoning by unspecified/unknown substances

Tab. 2. Classification of cases other than poisoning according to the cause of death.

\begin{tabular}{l} 
Cause of death \\
\hline Natural/internal \\
Accident \\
Suicide \\
Homicide \\
Undetermined
\end{tabular}

Tab. 3. Number of deaths and medicolegal autopsies and direct and indirect PAS related deaths and their percentage of all autopsies during the period of years 2007-2008.

\begin{tabular}{lcccccc}
\hline Year & $\begin{array}{c}\text { Number } \\
\text { of deaths }\end{array}$ & $\begin{array}{c}\text { Number of } \\
\text { ML autopsies }\end{array}$ & $\begin{array}{c}\text { Direct } \\
\text { deaths }\end{array}$ & $\begin{array}{c}\text { Indirect } \\
\text { deaths }\end{array}$ & $\begin{array}{c}\text { Total } \\
\text { Percentage of } \\
\text { PAS related } \\
\text { deaths }\end{array}$ \\
\hline 2007 & 11406 & 1142 & 15 & 21 & 36 & 3.15 \\
2008 & 11260 & 1130 & 23 & 25 & 48 & 4.25 \\
Total & 22666 & 2272 & 38 & 46 & 84 & 3.7 \\
\hline
\end{tabular}

PAS - psychoactive substances, ML - medicolegal

procedure by embedding in paraffin blocks and staining with haematoxylin-eosin. The sections were examined using a light microscopy.

For toxicological analyses, mostly samples of blood and urine were taken, but also samples of liver, kidney and stomach content, if necessary. The blood and urine specimens were analysed by gas chromatography for ethanol and other volatile substances content. The urine specimens were monitored for abused drugs and several prescription medicines classes using the method of group immunochromatographic semi-quantitative screening focused on the following substances: amphetamines, barbiturates, benzodiazepines, cannabinoids, cocaine, ecstase, methamphetamines, methadone, opiates, phencyclidine and tricyclic antidepressants. In the next step, the samples were examined by the method of high performance thin layer chromatography (HPTLC) in combination with densitometry (after the samples' preanalytical treatment by deproteination using the method by Faragó, followed by isolation of possibly present medicaments based on liquid-liquid extraction to diethylether and chlorophorm) and/or by gas chromatographymass spectrometry (GC-MS) technique (after the samples' preanalytical treatment based on the solid phase extraction (SPE)), used also for positive findings (HPTLC results) confirmation.

\section{Results}

In total, within the two-years 2007-2008, there were 84 fatalities related to psychoactive substances reported, which is $3.7 \%$ of all autopsies realized within the monitored period of time.

Out of that, 38 cases ( $45 \%$ ) belonged to the group of direct deaths (poisonings) and 46 cases $(55 \%)$ to the group with other cause of death. Summary of the number of cases, sorted by particular groups of deaths in the related years, as well as by number of deaths (6) and autopsies realized at the institutes of forensic medicine, is described in the Table 3.

Out of the total number of 84 cases, $70(83 \%)$ were related to men and $14(17 \%)$ to women. In the year 2007 , there were 36 cases $(43 \%)$ and in the year 2008, 48 cases $(57 \%)$. In the group of direct deaths, the most frequently detected substances were opioids themselves or in combination with other substances ( 20 cases, i.e. $53 \%$ ) and than psychoactive medicines (13 cases, i.e. $34 \%)$. Most of the cases $-24 \%$ - were related to the age category $30-34$

Tab. 4. Direct PAS related deaths cases autopsied in the period of years $2007-2008$ according to the detected substance(s), sex and age.

\begin{tabular}{|c|c|c|c|c|c|c|c|c|c|c|c|c|c|c|}
\hline PAS/ Age group & to 14 & $15-19$ & $20-24$ & $25-29$ & $30-34$ & $35-39$ & $40-44$ & $45-49$ & $50-54$ & $55-59$ & $60-64$ & $65-$ & Total (M/F) & Percentage \\
\hline Opioids only & - & - & - & - & $2 /-$ & - & - & - & $-/ 1$ & - & - & - & $3(2 / 1)$ & 7.9 \\
\hline Methadone only & $1 /-$ & - & - & - & - & - & - & - & - & - & - & - & $1(1 /-)$ & 2.6 \\
\hline PS incl. opioids & - & - & $2 /-$ & 4/- & $4 / 1$ & $3 /-$ & - & - & $1 /-$ & $1 /-$ & - & - & $16(15 / 1)$ & 42.1 \\
\hline PS excl. opioids & - & - & $1 /-$ & - & $-/ 2$ & - & $2 /-$ & - & - & - & - & - & $5(3 / 2)$ & 13.2 \\
\hline Psychoact. medicines & - & - & - & $1 / 1$ & - & $1 /-$ & $1 /-$ & $1 /-$ & - & $4 / 2$ & - & $1 / 1$ & $13(9 / 4)$ & 34.2 \\
\hline Total & $1 /-$ & - & $3 /-$ & $5 / 1$ & $6 / 3$ & $4 /-$ & $3 /-$ & $1 /-$ & $1 / 1$ & $5 / 2$ & - & $1 / 1$ & $38(30 / 8)$ & \\
\hline
\end{tabular}

PAS - psychoactive substances, $\mathrm{M}$ - male, F - female, PS - (poly)substances

Tab. 5. Indirect PAS related deaths cases autopsied in the period of years 2007 - 2008 according to the cause of death, sex and age.

\begin{tabular}{|c|c|c|c|c|c|c|c|c|c|c|c|c|c|c|}
\hline C. of death/Age group & to 14 & $15-19$ & $20-24$ & $25-29$ & $30-34$ & $35-39$ & $40-44$ & $45-49$ & $50-54$ & $55-59$ & $60-64$ & $65-$ & Total (M/F) & Percentage \\
\hline Natural/internal & - & - & - & $1 /-$ & $1 /-$ & $1 /-$ & $1 /-$ & $1 /-$ & - & $1 /-$ & $1 /-$ & - & $7(7 /-)$ & 15.2 \\
\hline Accident & - & - & $5 /-$ & $2 /-$ & $6 / 1$ & - & - & - & $1 /-$ & - & - & $1 /-$ & $16(15 / 1)$ & 34.8 \\
\hline Suicide & - & $1 /-$ & $5 / 1$ & $4 /-$ & $1 /-$ & - & $1 / 1$ & $1 /-$ & $1 /-$ & $-/ 1$ & - & $1 /-$ & $18(15 / 3)$ & 39.1 \\
\hline Homicide & - & $1 /-$ & - & - & $1 /-$ & - & $-/ 1$ & $-/ 1$ & - & - & - & - & $4(2 / 2)$ & 8.7 \\
\hline Undetermin. & - & - & - & - & - & - & - & - & - & $1 /-$ & - & - & $1(1 /-)$ & 2.2 \\
\hline Total & - & $2 /-$ & $10 / 1$ & $7 /-$ & $9 / 1$ & $1 /-$ & $2 / 2$ & $2 / 1$ & $2 /-$ & $2 / 1$ & $1 /-$ & $2 /-$ & $46(40 / 6)$ & \\
\hline
\end{tabular}

PAS - psychoactive substances, C. - cause, $\mathrm{M}$ - male, F - female 
Tab. 6. Indirect PAS related deaths cases autopsied in the period of years 2007 - 2008 according to the PAS detected, sex and age.

\begin{tabular}{|c|c|c|c|c|c|c|c|}
\hline PAS/ C. of death & Natural/internal & Accident & Suicide & Homicide & Undetermined & Total & Percentage \\
\hline Opioids & - & $3 /-$ & $3 / 1$ & - & - & $7(6 / 1)$ & 15.2 \\
\hline AMT/MAMT & $4 /-$ & $4 / 1$ & $5 / 1$ & $1 / 1$ & - & $17(14 / 3)$ & 36.9 \\
\hline Cocaine & - & $1 /-$ & - & - & - & $1(1 /-)$ & 2.2 \\
\hline Cannabis & - & $4 /-$ & $2 /-$ & - & - & $6(6 /-)$ & 13 \\
\hline Solvents & - & $1 /-$ & - & $1 /-$ & - & $2(2 /-)$ & 4.4 \\
\hline BZD & $2 /-$ & $2 /-$ & $4 / 1$ & $-/ 1$ & $1 /-$ & $11(9 / 2)$ & 23.9 \\
\hline Other medicines & $1 /-$ & - & $1 /-$ & - & - & $2(2 /-)$ & 4.4 \\
\hline
\end{tabular}

PAS - psychoactive substances, C. - cause, M - male, F - female, AMT - amphetamines, MAMT - metamphetamines, BZD - benzodiazepines

$\square$ direct deaths $\square$ undirect deaths $\square$ together

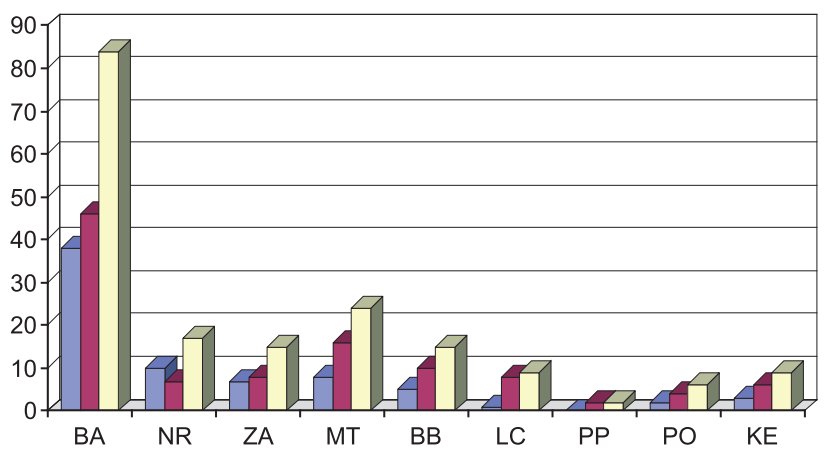

Fig. 1. The rate of cases of the psychoactive substances related deaths autopsied in Bratislava in comparison with other Slovak medicolegal workplaces.

years, whereas $50 \%$ of the cases were related to the age up to 34 years. A detailed summary of the cases reported within both years, sorted by the detected substances, sex and age, is listed in Table 4.

In the group of the indirect deaths, the most frequent category of death was suicide (18 cases, i.e. $39 \%$ ) and then accidents of various types ( 16 cases, i.e. $35 \%$ ). The most frequently found psychoactive substances were amphetamines and metamphetamines from the group of central nervous system stimulants ( 17 cases, i.e. $40 \%$ ) and benzodiazepines ( 11 cases, i.e. $24 \%$ ). Most of the cases $-24 \%$ - were reported in the category of 20-24 years of age. In the same category, within the age range up to 34 years, there were reported as many as $65 \%$ cases. A detailed summary of the cases reported during both years, sorted by cause of death, psychoactive substances detected, sex and age, is listed in the Tables 5 and 6 .

Evaluation of the data acquired from other regions of Slovakia has shown that the number of lethal cases autopsied in Bratislava represented more than $46 \%$ of the total 181 deaths related to psychoactive substances reported within the years 2007-2008 (Fig. 1) (7, 8a).

\section{Discussion}

In our study, we have found in absolute numbers the prevalence of deaths belonging to the group with the cause of death other than poisoning over the occurrence of deaths directly related to psychoactive substances application. An increase of the cases in the second year by $133 \%$ cannot be evaluated from the view point of statistical significance for the total number of cases and length of the monitored period of time. In the year 2007, the increase of the psychoactive substances related deaths number, after an intermediate reduction in the opening years of decennium, was reported by majority of the European States (9a).

The prevalence of men over women and a high incidence of deaths in younger age categories corresponds to data being reported for long term in Europe as well (10).

Among the psychoactive substances detected by toxicological analyses in the group of deaths caused by overdose in more than half of the cases dominated opiates and opioids. Within the group in the year 2007, we have noted a curious case of death related to the use of methadone by a 11-month-old male infant (11). Generally it is reported that in case of deaths related to psychoactive substances overdose, opiates and opioids are responsible for more than $85 \%(9 b)$.

In the group of deaths with a cause of death other than overdose, the most frequently found were substances from the group of central nervous system stimulants, namely amphetamines and metamphetamines. Generally, the number of their consumers is considered to be stable. The consumption of cocaine is, except in the European Union western countries, relatively low. In the year 2007, there were 500 deaths related to cocaine reported to the EMCDDA (9a). In our study, only one case of an indirect death related to cocaine was registered.

In both groups of death cases, the second most frequently found group of substances was the group of psychoactive medicaments with clear dominance of benzodiazepines.

The number of consumers of most frequently consumed/ abused psychoactive substance marihuana in Europe is on the decrease or stabilized. On contrary, in Slovakia its increase is reported $(9 \mathrm{c}, 8 \mathrm{~b})$. In our file, in the group of indirect deaths, cannabinoids were detected in only 6 cases, which was $13 \%$ of the total number in the group.

The problems of the psychoactive substances related deaths and the mortality of psychoactive substances consumers is one of the five key indicators monitored by EMCDDA. In past, practically up to the year 2004, in the countries of middle and eastern Europe the data had not been collected $(12,13,14)$. In Slovakia, a systematic monitoring of deaths related to psychoactive substances for EMCDDA has begun in the year 2005 (data 2004) (15). Its method, development and results are subject of other more extensive paper planned to be published in the near future. 
In this paper, the reported number of psychoactive substances related deaths on the territory of Bratislava and Trnava districts and on the whole territory of Slovakia within the 2-years period of time is relatively low compared to the total number of deaths and autopsied cases (7c, 8a). However, that should not be the reason for neglecting the problem of drug abuse. The proved reality indicates inaccuracies and failures in the monitoring of prevalence of the psychoactive substances related deaths in population. Such assumption is supported also by a disproportion in number of the deaths reported by workplaces of forensic medicine in Bratislava versus others in remaining parts of Slovakia. To explain the found status, several options are available. At first, it is possible to state that the found numbers reflect the reality that in territory of the Slovakia capital city and within its vicinity is concentrated the highest number of psychoactive substances consumers and/or abusers (8c). Another reasons are practical, involving particularly economic demands of toxicological examinations. Such relevant examinations are not sufficiently requested at autopsies of deaths, predominantly in the group with the cause of death other than poisoning, where the direct cause of death is generally evident. This situation results also from the unequal standard of toxicological laboratories, which points on urgent need to define conception of the Slovak forensic toxicology and to finish a creation of toxicological laboratories network (16). The recent situation can be improved also by gaining financial resources for purchasing reagents in the amounts essential for qualitative and quantitative analysis of psychoactive substances in the samples isolated from dead bodies and for determination of their relationship to death.

Last but not least, the human factor in data collection should be considered. It is assumed that there are workplaces where the mentioned types of autopsies were performed, but not reported. However, it is not assumed that significant differences between actual and reported numbers of deaths exist.

\section{References}

1. Steentoft A, Teige B, Holmgren $\mathbf{P}$ et al. Fatal poisonings in young drug addicts in the Nordic countries: a comparison between 1984-1985 and 1991. Forensic Sci Int 1996; 78: 29-37.

2. Vicente J, Frost N, Hartnoll R. EMCDDA standard protocol for the EU member states to collect data and report figures for the key indicator drug-related deaths by the standard reitox tables. Lisbon, European monitoring centre for drugs and drug addiction, 2002; 37.

3. Sidlo J. Psychoactive substance-related deaths in road traffic accidents in Slovakia between 2000 and 2007. Bratisl Med J 2009; 110: 468-471.

4. Kuruc R, Sidlo J, Valent D, Mlynar J, Murarikova B. The responsibility of alcohol-impaired road users in fatal road traffic accident. Bratisl Med J 2009; 110: 802-806.

\section{5. http://www.zbierka.sk/}

\section{6. http://www.portal.statistics.sk/}

7. Sidlo J. Drug related deaths and mortality of drug users. In: Kastelova E (ed): National report on drugs in Slovakia for the year 2007. Bratislava, National monitoring centre for drugs, 2008: 56.

8. Sidlo J. Drug related deaths in 2008. 2009 National report (2008 data) to the EMCDDA by the Reitox National Focal Point. Bratislava, National monitoring centre for drugs, 2009: 79 (a), 25 (b), 9 (c).

9. Annual report 2009. The state of the drugs problem in Europe. European monitoring centre for drugs and drug addiction. Luxembourg, Publications office of the European union, 2009, ISBN 978-92-9168-384-0: 86 (a), 85 (b), 45 (c).

10. Annual report 2008. The state of the drugs problem in Europe. European monitoring centre for drugs and drug addiction. Luxembourg, Office for official publications of the european communities, 2008, ISBN 978-92-9168-324-6: 82.

11. Sidlo J, Valuch J, Ocko P, Bauerova J. Fatal methadone intoxication in a 11-month-old male infant. Soud Lek 2009; 54: 23-25.

12. Hartnoll R. Multi-city study: Drug misuse trends in thirteen European cities. Strasbourg, Council of Europe, Publishing and documentation service, 1994: 49.

13. Multi-city network eastern Europe drugs and addiction. Strasbourg, Council of Europe publishing, 1997: 410.

14. Karlovsek ZM. Illegal drugs-related fatalities in Slovenia. Forensic Sci. Int. 2004; 146S: S71-S75.

15. Okruhlica L, Sidlo J. The health implications and consequences of drug use. In: Kissova L et al. (eds): The state of drug addiction and drug control in the Slovak Republic. 2005 Report. Bratislava, National monitoring centre for drugs, 2005: 111.

16. http://www.udzs.sk/

Received April 7, 2010. Accepted September 20, 2011. 